\title{
Formation of Two-Dimensional Network of Organic Charge- Transfer Complexes at the Air-Water Interface
}

\author{
Arpan Bhattacharyya ${ }^{1,2}$, Milan K Sanyal ${ }^{1,2^{*}}$, Umesha Mogera ${ }^{2}$, Subi J George ${ }^{2}$, Shikha \\ Dhiman $^{2}$, Giridhar. U. Kulkarni2,3, Philippe Fontaine ${ }^{4}$ \\ ${ }^{1}$ Saha Institute of Nuclear Physics, 1/AF Bidhannagar, Kolkata 700 064, India, \\ ${ }_{2}^{2}$ Jawaharlal Nehru Centre for Advanced Scientific Research, Jakkur, Bangalore 560064, India \\ ${ }^{3}$ Centre for Nano and Soft Matter Sciences, Jalahalli P.O., Bangalore, 560013, India \\ ${ }^{4}$ SOLEIL Synchrotron, L’Orme des Merisiers, Saint-Aubin - BP48, 91192 GIF-sur-YVETTE CEDEX, \\ France
}




\section{Contents}

1. Synthesis of DMV and CS molecules 3

2.Formation of DMVCS monolayer 3

3. GID experiment and collected data $\quad$ 4-7

$\begin{array}{lc}\text { 4. AFM Imaging of DMVCS film } & \text { 7-9 }\end{array}$ 


\section{Synthesis of DMV-CS and DMV molecule:}

The synthesis of DMV and CS molecules has already been as referred in the main text. In brief, CS was synthesized by a twofold oxidative benzogenic Diels-Alder reaction of perylene with $\mathrm{N}$ ethyl maleimide and subsequent hydrolysis with $\mathrm{KOH}$ in methanol. DMV was synthesized from 4, 4'-bipyridine by controlled reaction on one nitrogen with dodecyl bromide to give mono pyridinium ion and followed by treating the with methyl iodide to amphiphllic dicationic bipyridine (DMV).

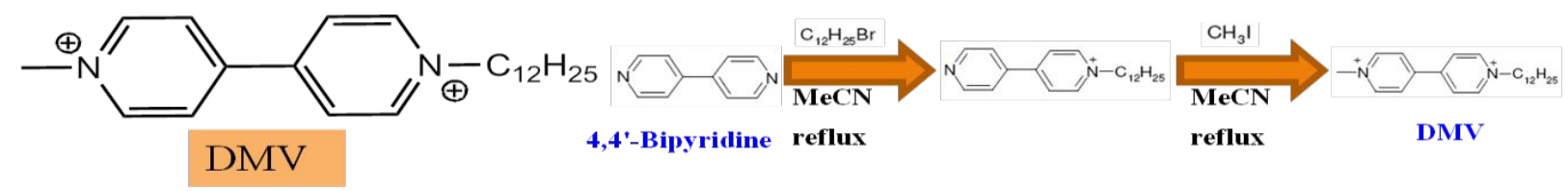

\section{Dodecyl Methyl Viologen}<smiles>O=C([O-])c1c(C(=O)[O-])c2ccc3ccc4c(C(=O)[O-])c(C(=O)[O-])c5ccc6ccc1c1c6c5c4c3c21</smiles>

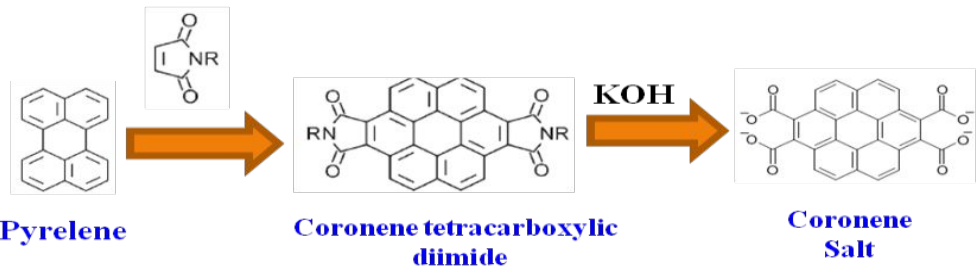

CS was synthesized by a twofold oxidative benzogenic Diels-Alder reaction of perylene with $\mathrm{N}$-ethyl maleimide and subsequent hydrolysis with KOH in methanol

Coronene Tetracarboxylate Potassium Salt

K.Rao et.al Angew. chem int ed 2010, 49, 4218-4222

Schematics of synthesis

\section{Monolayer Formation:}

The molecular structures of DMV and CS and monolayer isotherm are indicated in Figure 1(a) of the main text and in the above schematics. For the DMV Monolayer formation on the water surface, $10 \mathrm{ml}$ of $1 \mu \mathrm{M}$ DMV solution in chloroform was sonicated for $15 \mathrm{~min}$. After complete dispersion of the solute it was dispersed drop by drop on the water surface of the trough. DMVCS monolayer was formed by adding CS to the trough water to reach $1 \mu \mathrm{M}$ concentration before spreading DMV solution over the water surface. One hour waiting time was given before collecting X-ray GID data. 


\section{GID Measurements:}

We have used the grazing incidence diffraction (GID) technique to probe 2D ordering of molecular monolayers at the air-water interface as the penetration of X-ray beam can be reduced drastically by using angle of incidence close to the critical angle of the water surface. It should be mentioned here that GID studies from liquid surface required special spectrometers to bend the X-ray beam down to control grazing angle of incidence. The in-situ GID measurements with varying surface pressure were conducted in a Langmuir trough at SIRIUS beamline in the synchrotron SOLEIL, France. The beam energy was $8 \mathrm{keV}$ and all the data was taken using a Pilatus (Dectris, Switzerland) detector by scanning in the in-plane direction with the beam incidence angle $\theta \mathrm{i}=2$ milliradians. The 2D image was generated by integrating subsequent window of the original raw 2D file of the detector (Figure 1(b) of the main text). GID data were taken as a function of surface pressures by controlling the separation between the trough barriers. The data were taken from the DMV-monolayer with and without having CS in the subphase. Some of the data taken at various surface pressure are shown below.

\section{Collected data in the detector and extracted GID line-profile}

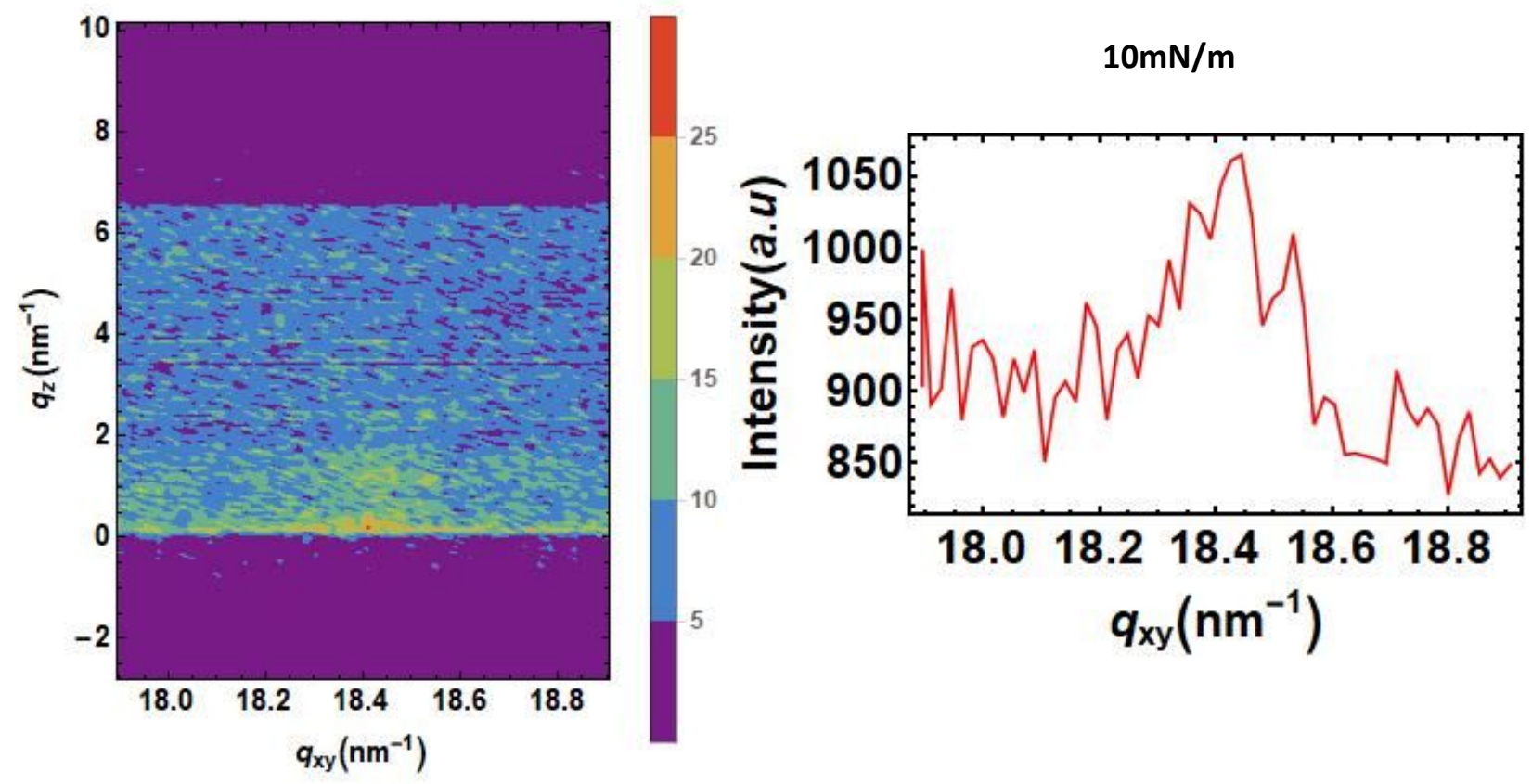

Figure S1. Detector data and 1D reduction of $10 \mathrm{mN} / \mathrm{m}$ DMVCS data showing onset of Charge Transfer (C-T) ordering. 


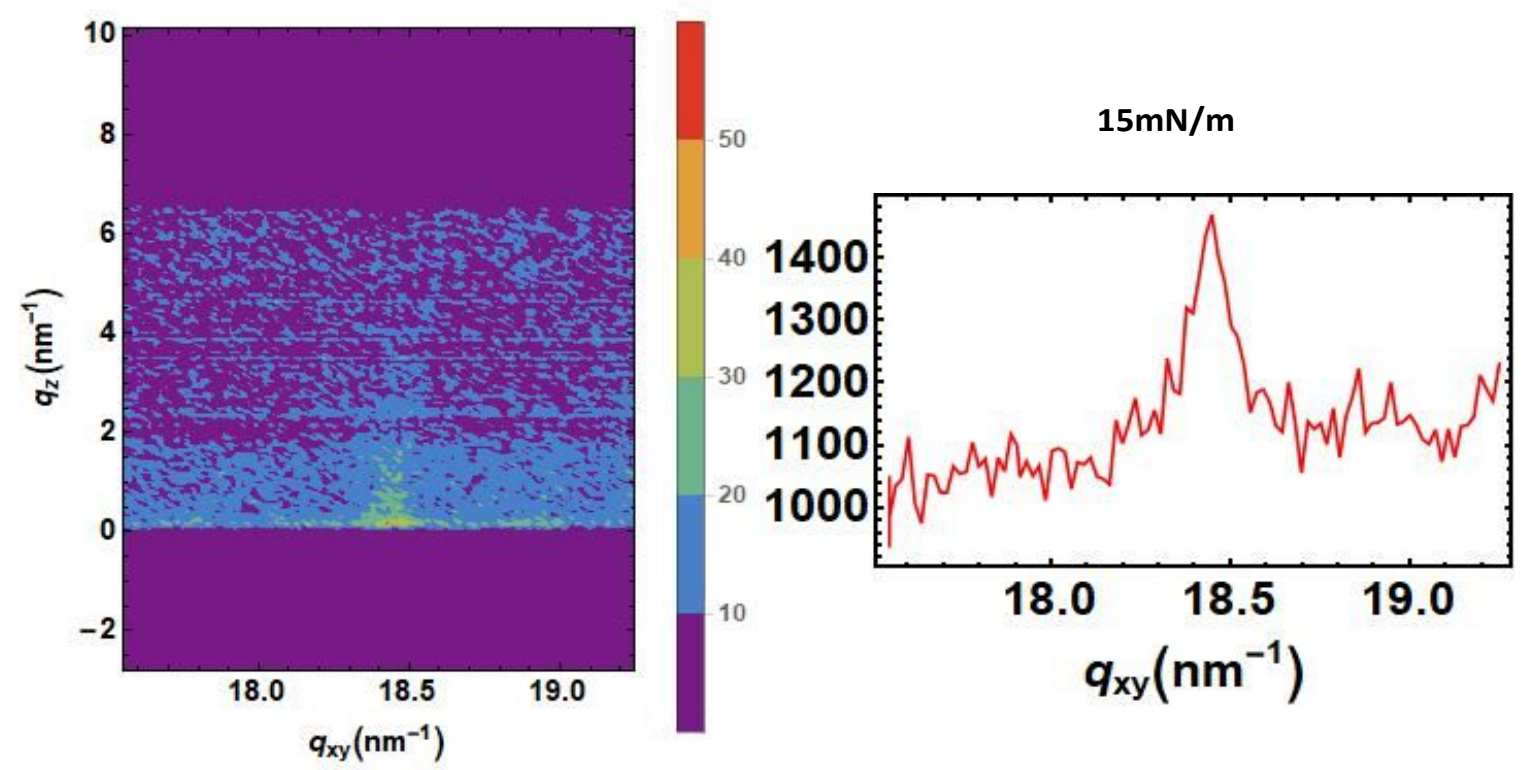

Figure S2. Detector data and 1D reduction of $15 \mathrm{mN} / \mathrm{m}$ DMVCS data showing stronger C-T peak

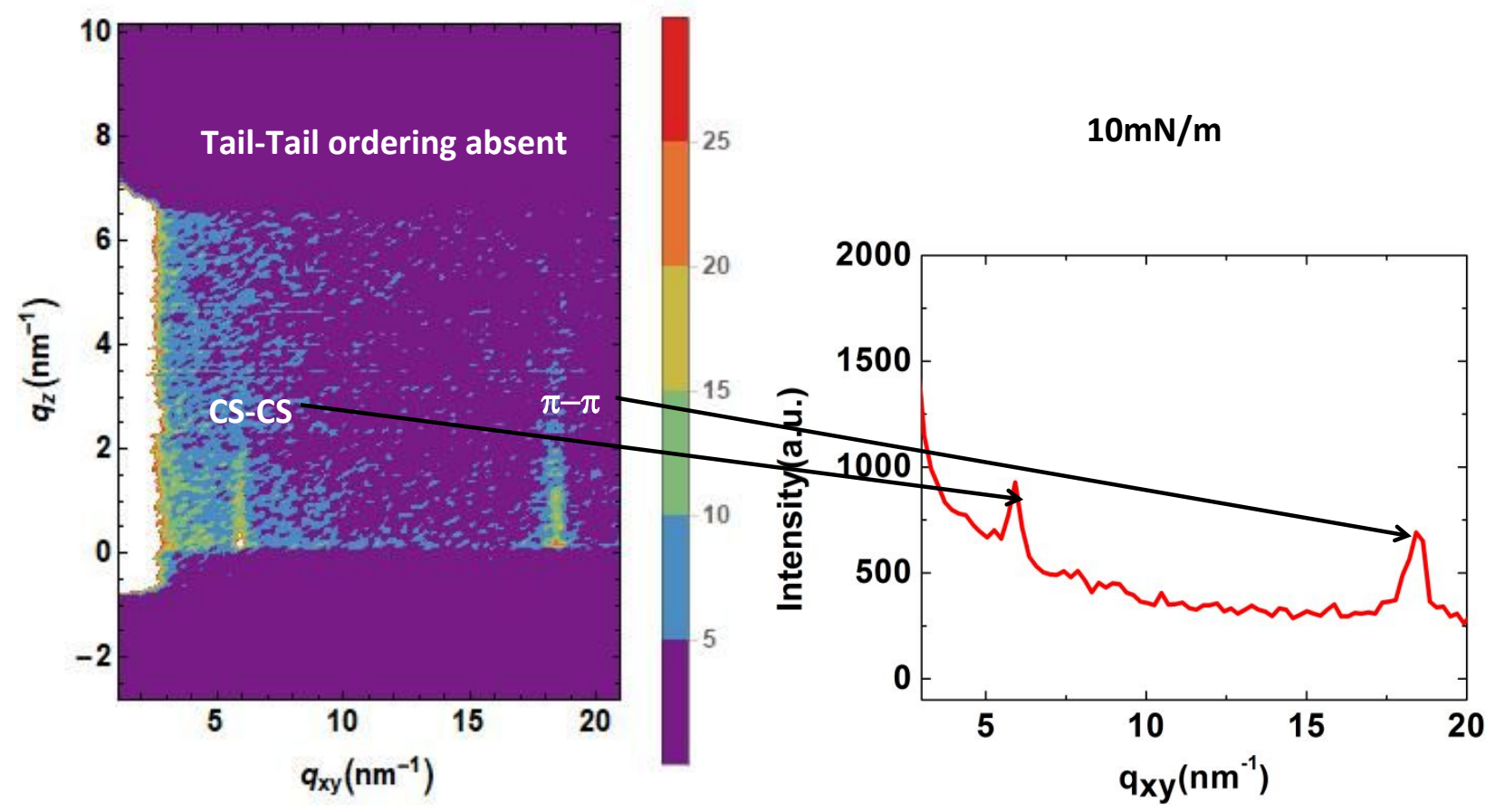

Figure S3. Long Detector scan and 1D extracted data of $10 \mathrm{mN} / \mathrm{m}$ DMVCS data showing stronger CT peak and CS-CS ordering together 

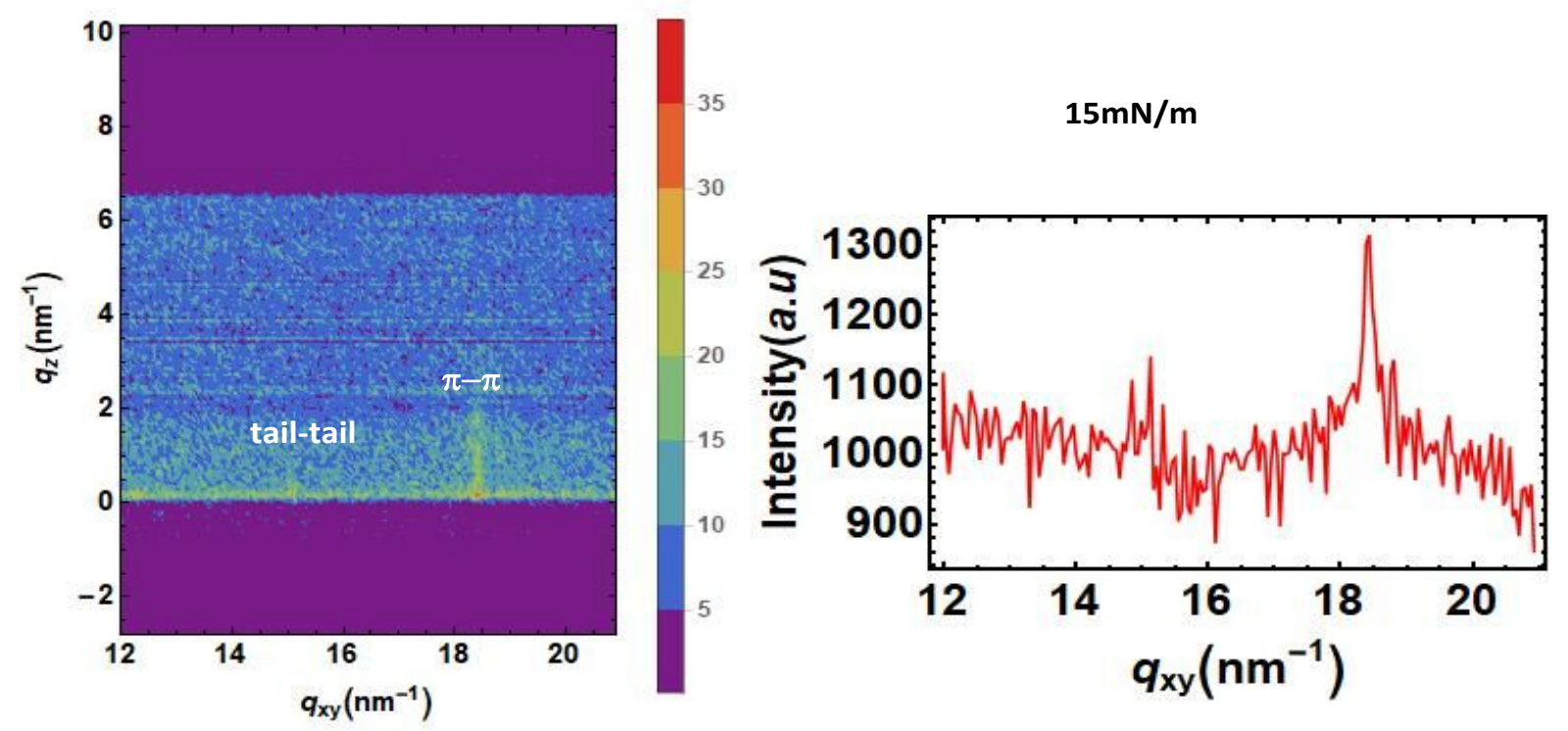

Figure S4 Long Detector scan and 1D extracted data of 15mN/m DMVCS data showing CT peak and tailtail (T-T)ordering together
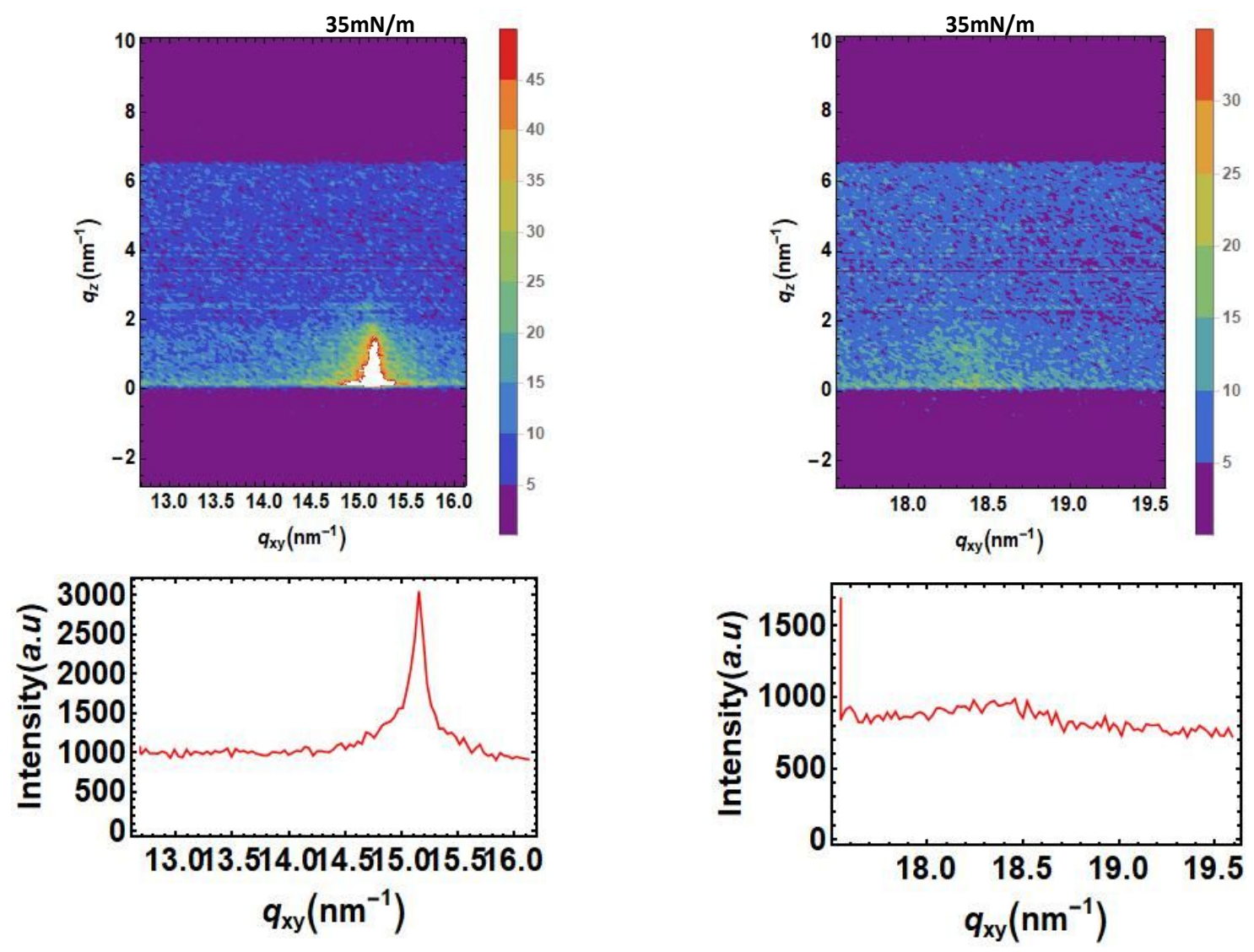

Figure S5. Detector data and 1D reduction of $35 \mathrm{mN} / \mathrm{m}$ DMVCS data showing almost no CT ordering and showing strong T-T ordering 

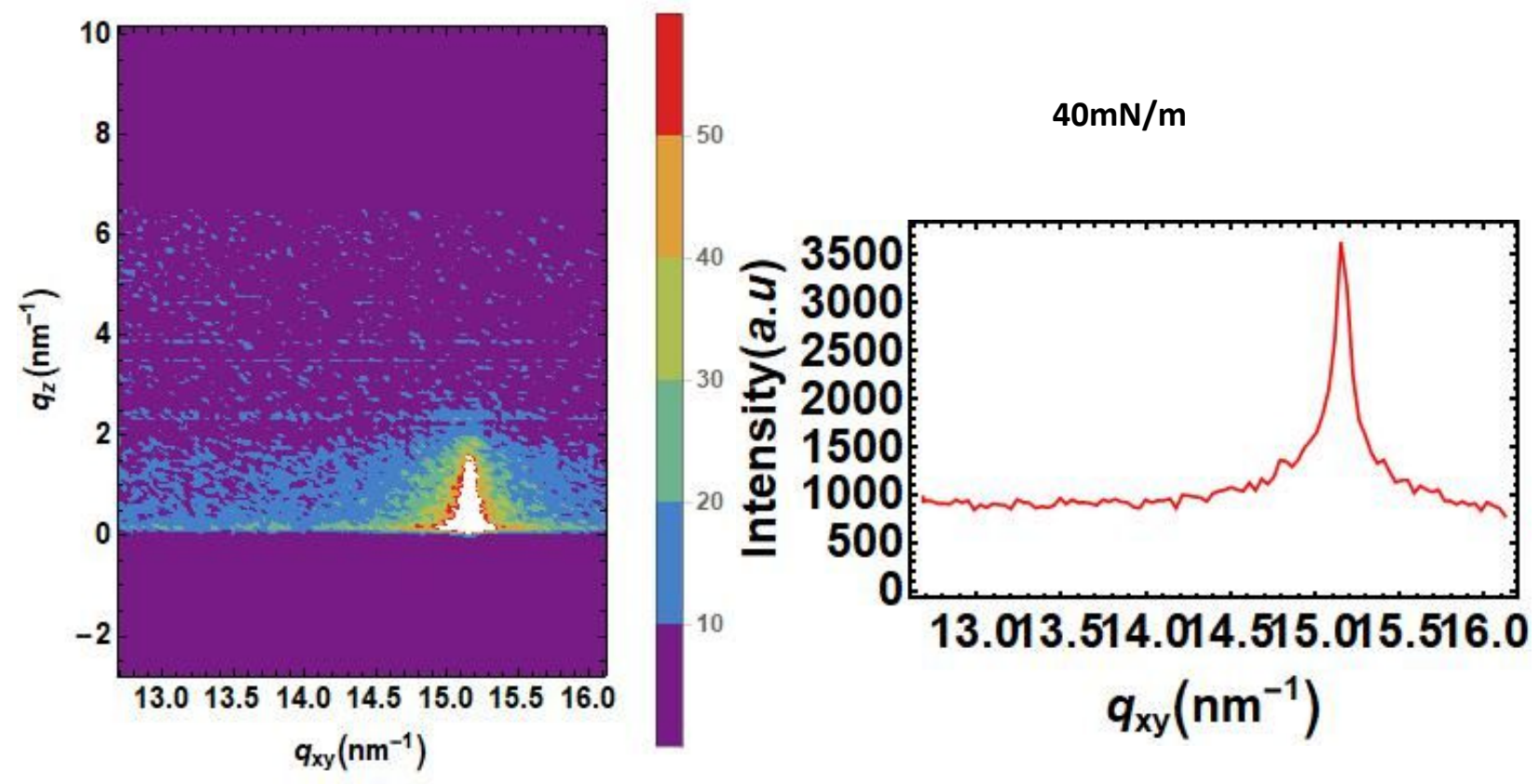

Figure S6. Detector data and 1D reduction of $40 \mathrm{mN} / \mathrm{m} \mathrm{DMVCS}$ data showing strong T-T ordering

\section{AFM Imaging of LB film on Si substrate:}

Hydrophilic fuctionalization of Si Substrate (RCA Cleaning): The Si substrate (Siltronix P-Type wafers) were cleaned by sonicating in Chloroform (Merck), Isopropyl Alcohol (IPA) (Merck) and Acetone (Merck) for $5 \mathrm{~min}$ each, then cleaned with IPA again and dried. The wafers were transferred to a beaker containing Water $\left(\mathrm{H}_{2} \mathrm{O}\right)$ (Millipore $\left.\rho=18 \mathrm{M} \Omega\right)$, Hydrogen Peroxide $\left(\mathrm{H}_{2} \mathrm{O}_{2}\right)$ (Merck Reagent Grade) and Ammonia $\left(\mathrm{NH}_{4} \mathrm{OH}\right.$ ) (Merck Reagent Grade) in the ratio $2: 1: 1$ and was heated for $12 \mathrm{~min}$ at $80^{\circ} \mathrm{C}$. Later the substrates were further treated under UV lamp for 30 minutes to enhance the hydrophilicity of the substrates.

LB Film Deposition: The LB film deposition was carried out using the KSV-NIMA 5000 LB deposition system. For the DMV Monolayer formation on the water surface $10 \mathrm{ml} 1 \mu \mathrm{M}$ DMV solution in chloroform was sonicated for $15 \mathrm{~min}$. After complete dispersion of the solute it was drop by drop dispersed on the water surface of KSV-NIMA LB trough. DMV-CS CT complex amphiphile monolayer was formed by initially adding CS to the LB trough water to $1 \mu \mathrm{M}$ concentration then repeating the process of DMV monolayer formation with an additional waiting time after the addition DMV dissolved in chloroform to $1 \mathrm{hr}$ so that the CT complex formation have enough time to take place.

The hydrophilic substrate was immersed below the water surface at zero trough pressure before the addition of DMV monolayer and was retracted rate of $2 \mathrm{~mm} / \mathrm{min}$ after reaching the desired pressure. 
AFM Imaging: AFM imaging was done on a dilnnova SPM (Veeco, USA) using Si probes (spring constant $40 \mathrm{~N} / \mathrm{m}$ ) in tapping mode. We have shown typical AFM images of DMV and DMV-CS monolayers in the main text. These films were transferred to hydrophilic silicon substrate at $25 \mathrm{mN} / \mathrm{m}$ pressure. The transferred monolayer of DMV-CS after the collapsepressure show the formation of fiber-like structures of diameter 6-7 nm (refer Figure 4(c) in the main text), similar to that of the reverse micelles found in the solution-grown fibers. The length of the nanofibers obtained from the collapsed monolayer was found to be around $100 \mathrm{~nm}$. 